\section{The thyroid axis just got more complicated}

\author{
Fredric E. Wondisford
}

Endocrinology and Metabolism Section, Department of Medicine The University of Chicago, 5841 S. Maryland Avenue, Chicago, Illinois 60637, USA.

Phone: (773) 702-6217; Fax: (773) 834-0486; E-mail: fwondisf@medicine.bsd.uchicago.edu.

J. Clin. Invest. 109:1401-1402 (2002). doi:10.1172/JCI200215865.
As a first-year medical student, you could count on the simplicity and elegance of the thyroid axis, because all its details had apparently been elucidated. Hypothalamic thyrotropin-releasing hormone (TRH) promoted the synthesis, release, and glycosylation of pituitary thyrotropin, thyroid-stimulating hormone (TSH), which in turn acted on the thyroid follicle, leading to the synthesis and release of thyroid hormones $\left(\mathrm{T}_{4}\right.$ and $\left.\mathrm{T}_{3}\right)$. Furthermore, precise control of the axis was maintained by thyroid hormone negative feedback at both the hypothalamic and pituitary levels (Figure 1). Now, a new pituitary hormone, termed thyrostimulin, may change our established view of this axis.

TSH, follicle-stimulating hormone (FSH), luteinizing hormone (LH), and chorionic gonadotropin (CG) are a family of heterodimeric pituitary and placental glycoprotein hormones that share a common $\alpha$ subunit (1). Their biological specificity at either the thyroid or the gonad is conferred by a unique $\beta$ subunit that is noncovalently linked to the common glycoprotein $\alpha$ subunit. All of these hormones have been purified by classical means, and their biological activities established by in vivo assays.

Nakabayashi et al. in this issue of the JCI (2) describe a novel heterodimeric glycoprotein hormone containing two unique subunits, which they term A2 and B5. These investigators identified A2 and B5 in a homology search of the human genome (GenBank). In a nearly simultaneous paper in another journal, the same group describes orthologs of these subunits found in diverse species (3). A2 and B5 share only modest amino acid similarity with the related glycoprotein hormone subunit family members, but they contain all of the cysteine residues required to form the characteristic cysteine knot structure found in these and other growth factors, including PDGF and TGF- $\beta$.
The authors show here that A2 interacts strongly with FSH- $\beta, \mathrm{CG}-\beta$, and B5 in a yeast two-hybrid assay (2). After determining that the $\mathrm{A} 2 / \mathrm{B} 5$ pair can stimulate cAMP production via the TSH receptor, Nakabayashi and colleagues pursued the thyrotropic activity of A2/B5 in greater detail.

\section{Patterns of expression and sites of action}

A2, also referred to as GPA2, is ubiquitously expressed and is 35\% identical at an amino acid level to the common glycoprotein $\alpha$ subunit (GPA1). A2 expression is limited to the pituitary, where TSH, FSH, and LH are synthesized, and the placenta, where CG is synthesized. B5 is approximately $30 \%$ identical at an amino acid level to TSH- $\beta$, FSH- $\beta$, or LH- $\beta$. Its pattern of expression is more restricted than that of A2 and includes the brain, pituitary, thyroid, ovary, and heart. Nakabayashi et al. clearly demonstrate the coexpres-

\section{Commentary}

See related article, pages 1445-1452. sion of $\mathrm{A} 2$ and $\mathrm{B} 5$ in the anterior pituitary (2), although the precise cell types expressing these subunits have not yet been identified.

Mammalian cells transfected with expression vectors for A2 and B5 secrete a heterodimeric glycoprotein hormone, which the authors term thyrostimulin. This novel hormone is active at the TSH but not the FSH or $\mathrm{LH}$ receptor. Indeed, as judged by its ability to stimulate cAMP production, thyrostimulin is as active as bovine TSH (the most active, purified wildtype preparation available) and is approximately sevenfold more active than either pituitary-derived or recombinant human TSH preparations.

The authors are appropriately cautious about the role of thyrostimulin in normal thyroid physiology. For example, they have yet to demonstrate whether thyrostimulin circulates in the bloodstream or is responsive to thyroid hormone negative feedback

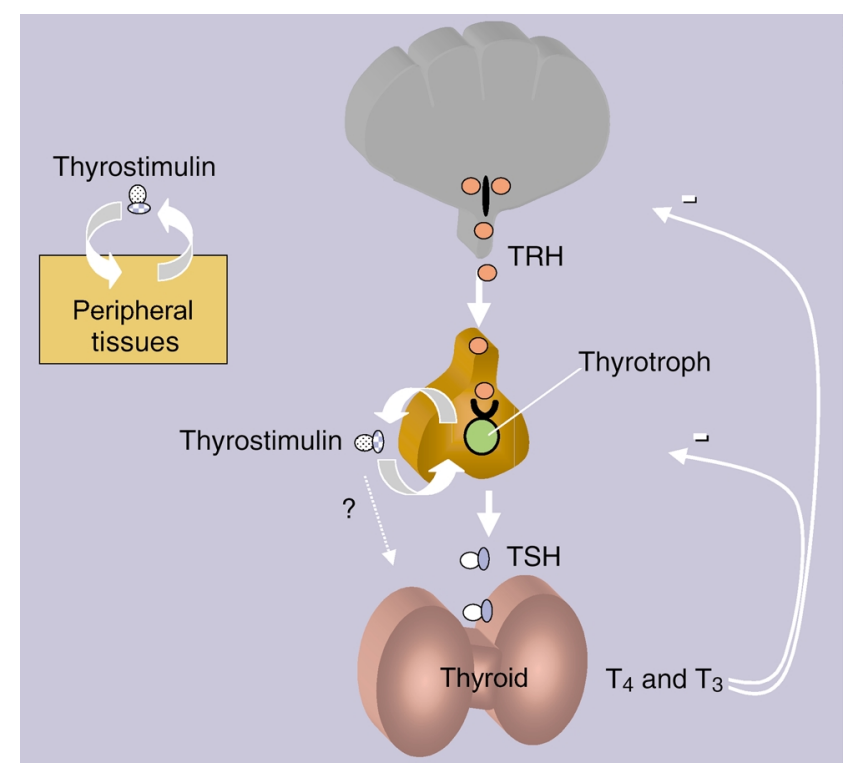

Figure 1

The role of thyrostimulin in the thyroid axis and periphery. Thyrostimulin, a heterodimeric glycoprotein hormone, is synthesized in the pituitary and other peripheral tissues. It is suggested to act in a paracrine fashion at these sites. A role for thyrostimulin at the thyroid is purely speculative. 
control. Given its apparently broad expression pattern, thyrostimulin may not function or be regulated in the same manner as TSH. Based on the finding of hypothyroidism in patients with TSH- $\beta$ mutations - in whom thyrostimulin expression should be normal - Nakabayashi and colleagues suggest that peripherally produced thyrostimulin acts in a paracrine manner, with little effect on thyroid function (2). However, most of these patients have measurable, albeit low, concentrations of $T_{4}$ and $T_{3}$ in the serum (4-8), supporting the longstanding observation that primary forms of hypothyroidism may be clinically more severe than central forms. TSH-independent production and secretion of $\mathrm{T}_{4}$ and $\mathrm{T}_{3}$ from the thyroid have been attributed to constitutive activity of the unbound TSH receptor. However, if thyrostimulin is secreted in sufficient quantities from the pituitary, this novel hormone could account for the residual stimulation of the thyroid in patients with central hypothyroidism caused by TSH deficiency.
Another important observation in the present work (2) is the peripheral expression of both A2 and B5, notably coexpression of both subunits in tissues that, like the thyroid, have been reported to express the TSH receptor (9-11) - the brain, thyroid, heart, and oviduct (adipose tissue and orbital fibroblasts were not examined here). Does thyrostimulin play a role in extrathyroidal stimulation of the TSH receptor? If so, does thyrostimulin control metabolism in cardiac or adipose tissue, or does it modify the immune response of the orbital fibroblasts in Graves disease or in the controversial disorder termed Hashimoto encephalopathy? For now, there are many more questions than answers, but the findings of Nakabayashi et al. (2) have clearly expanded our view of what constitutes normal thyroid physiology.

1. Cohen, R.N., Weintraub, B.D., and Wondisford, F.E. 2000. Factors that control thyroid function: thyrotropin. In The thyroid: a fundamental clinical text. L.E. Braverman and R.D. Utiger, editors. Lippincott Williams \& Wilkins. Philadelphia, Pennsylvania, USA. 202-233.

2. Nakabayashi, K., et al. 2002. Thyrostimulin, a heterodimer of two new human glycoprotein hormone subunits, activates the thyroid-stimulating hormone receptor. J. Clin. Invest. 109:1445-1452. doi:10.1172/JCI200214340.

3. Hsu, S.Y., Nakabayashi, K., and Bhalla, A. 2002. Evolution of glycoprotein hormone subunit genes in bilateral metazoa: identification of two novel human glycoprotein hormone subunit family genes, GPA2 and GPB5. Mol. Endocrinol. In press.

4. Miyai, K., Azukizawa, M., and Kumahara, Y. 1971 Familial isolated thyrotropin deficiency with cretinism. N. Engl. J. Med. 285:1043-1048.

5. Dacou-Voutetakis, C., Feltquate, D.M., Drakopoulou, M., Kourides, I.A., and Dracopoli, N.C. 1990. Familial hypothyroidism caused by a nonsense mutation in the thyroid-stimulating hormone $\beta$ subunit gene. Am. J. Hum. Genet. 46:988-993.

6. Medeiros-Neto, G., et al. 1996. A circulating, biologically inactive thyrotropin caused by a mutation in the beta subunit gene. J. Clin. Invest. 97:1250-1256.

7. Doeker, B.M., Pfaffle, R.W., Pohlenz, J., and Andler, W. 1998. Congenital central hypothyroidism due to a homozygous mutation in the thyrotropin $\beta$-subunit gene. J. Clin. Endocrinol. Metab. 83:1762-1765.

8. Heinrich, C., et al. 2000. Congenital central isolated hypothyroidism caused by a homozygous mutation in the TSH- $\beta$ subunit gene. Thyroid. 10:387-391.

9. Crisanti, P., et al. 2001. The expression of thyrotropin receptor in the brain. Endocrinology. 142:812-822.

10. Sellitti, D.F., et al. 1997. Differential expression of thyrotropin receptor mRNA in the porcine heart. Thyroid. 7:641-646.

11. Bell, A., et al. 2000. Functional TSH receptor in human abdominal preadipocytes and orbital fibroblasts. Am. J. Physiol. Cell Physiol. 2000. 279:C335-C340. 